\title{
Schema Evolution in Object Databases by Catalogs
}

\author{
Anne Pons \\ Département d'Informatique \\ Université du Québec à Montréal \\ C.P. 8888, Succursale Centre-ville \\ Montréal (Québec), Canada \\ H3C $3 P 8$, \\ voice: (514) 987-3000x6982, fax: (514) 987-8477 \\ e-mail: pons.anne@uqam.ca \\ Rudolf K. Keller \\ Département d'Informatique et de Recherche Opérationnelle \\ Université de Montréal \\ C.P. 6128, Succursale Centre-ville \\ Montréal (Québec), Canada \\ H3C $3 \mathrm{~J} 7$ \\ voice: (514) 343-6782, fax: (514) 343-5834 \\ e-mail:keller@iro.umontreal.ca
}

\begin{abstract}
We are concerned by schema evolution in Object Oriented Databases (OODB) that is processed by a modification on the classes on the schema. We present a new categorization of the different modifications in three categories : primitive, composite and complexe modifications. On this basis, we propose a method by decomposition for addressing conceptual schema evolution: real-life, complex schema modifications are solved by decomposition into simpler, well-known and controlled modifications, called composites, which in turn may be reduced to so-called primitives. A key step in making this approach practical, is the provision of two catalogs: one for the primitives based on a sound object model and one for the composites. Such catalogs raise the level of abstraction, further reuse and are prerequisites for effective tool support. In this paper, we define the three types of schema modifications, describe these catalogs, put them into the context of our decomposition approach and provide a process for schema evolution.
\end{abstract}

Keywords: object-oriented database; schema evolution; primitive, composite, complex modification; decomposition; Object Database Management Group (ODMG) .

\section{Introduction}

The conceptual schema of an Object-Oriented Database (OODB) is subject to modifications, as applications and their environment evolve. Modifications might be necessitated by the acquisition of new knowledge, by new prerequisites and constraints, or by the growth of applications. They tend to be quite frequent in the object-oriented context. The domains of applications (CAD/CAM or multimedia) that use OODB, are in general immature and young and this fact leads to often change the structure of the data, captured by the OODB schema.

Conceptual schema evolution is often complex. We are confronted to two main problems that we call the applicability problem(does the underlying OODB support the desired evolution and how realize it) and the conformity problem(are the evolved schema, datas and application conform to the required evolution?). This last problem involves not only to preserve the structural and behavioral consistency of the conceptual level (conceptual reorganization), but also requires the physical rearrangement of data (physical reorganization), and the adaptation of the applications to the underlying, evolved schema (external reorganization). The conformity ensures the stability of the system after the evolution.

In this paper, we propose a method for addressing conceptual schema evolution in object-oriented database system, based on a new categorization of the possible modifications a schema could undergo. We have adopted an approach of 
decomposition to solve -according to the applicability and conformity problems- arbitrary schema modifications at three levels of complexity: "real-life", complex schema modifications are solved by decomposition into simpler, well-known and controlled modifications, called composites, which in turn may be decomposed into so-called primitives. Primitive modifications are the modifications that should be supported by the underlying OODB and for which the conceptual, physical and external reorganizations are controlled. We have determined a catalog of primitive modifications which is based on an explicit definition of the notion of primitive. This catalog contains the description of the primitives including the reorganizations to be done for each of them. On this basis, we have defined the composite modifications and we propose a second catalog containing the most typical and the most frequently occurring composite modifications. With this catalog, our main objective is to provide a higher level of abstraction for schema evolution than the primitive level. We focus in this paper on these catalogs which present and categorize schema modifications in a new light. In addition, a scenario is presented for the process of schema evolution, using these two catalogs. Our work is a prerequisite for the automation of the high-level modifications by a tool, in order to aid and assist the task of evolution.

Primitive modifications are mentioned in different works. They are supported by advanced OODBs like ORION [4] which authorizes a lot of primitive modifications, expressed with a classification. This classification is often re-used [10, 20, 24, 25]. Tresch and Scholl [22] suggest to classify a modification depending if it preserves, augments or reduces the set of instances during transformation. The catalog of primitives that we propose is complete according to the explicit definition of primitive modifications we have given. It provides a stable and solid base for the more complex modifications. It allows to find a decomposition for the composites of our second catalog and to solve the problems of schema evolution for these modifications. We have validated this catalog by studying different work in schema evolution domain[2, 3, 11, 13, 14, 21], in schema integration domain[8] and in refactoring domain[17] where several modifications are discussed. We provide the results of the comparisons with the catalog of composites.

Before providing an overview of the three levels of modifications in section 3, we first present in section 2 the definition of a primitive modification. This helps the presentation of our work. In section 4, we proceeds with the catalog of the composite modifications. Finally, in section 5, we present the process of schema evolution, followed by a conclusion.

\section{Primitive modifications}

The primitive modifications are conceptual schema modifications at the lowest level of complexity for schema evolution that an OODB offers in its evolution functionality. Controlling them will help us to resolve modifications at higher levels of complexity. The set of primitives of a system depends on its underlying object model. To be independent from any OODB system, we use as object model reference the ODMG'93 model [1]. The ODMG makes the distinction between type and class. Types have multiple implementations and class is a couple (type, implementation). We consider types as parametrizable classes (in terms of implementation of methods and private data/methods). We make the simplifying assumption that we do not have parametrizable classes. The systems, in general, do not support parameterized implementations. The model includes the Relationship concept. ODMG enriches the Class concept with the notions of extension and key. Keys are attributes of the class whose values uniquely identify the instances. In addition, the model allows multiple inheritance and doesn't have an explicit common root to the classes. The catalog of primitives we have presented in $[18,19]$ is structured according to the well-known object-oriented concepts: class, inheritance, attribute, method and relation that we find in the ODMG. They represent the different atomic parts of a schema that can be modified. Each of them is described by characteristics given by the ODMG. These concepts lead to the definition of our primitive modifications that we recall here :

\section{Definition:}

Given a concept, a primitive modification can be one and only one of the three types of modifications:

- create one and only one element of the concept in the schema

- remove one and only one element of the concept from the schema

- change one and only one element of the concept in the schema, according to the characteristics of the concept.

To ensure the schema consistency after a primitive modification, invariants have been defined expressing the conditions under which the consistency is preserved. A schema has to be conform with the object model regarding these invariants. If the consistency is not preserved by a conceptual modification, we give rules as recipes to keep the schema consistency. For example, the primitive "Remove a class" could violate the invariant 2 if the class is referenced somewhere whereas it 
doesn't exist anymore. We have not seen in detail the behavioral consistency and we don't focus on the reorganization of the application. Some work exist already $[15,17,23]$. For the physical reorganization, we claim that any primitive assures no loss or minimal loss of data to preserve the existing information as much as possible. ([21] says that this point is part of the definition of schema evolution.) We have defined different data transformations to update data without losing information. Since an instance has a class of membership and a set of values corresponding to inherited attributes or relationships, we propose the following tranformations of an instance :

- change its membership (we say reclassify) for a direct sub-class;

- compute the value of an attribute whose type doesn't reference a type class, knowing the old value before evolution;

- create, remove or change instances, values of an attribute or participants in a relationship, knowing the old state of this element if it is modified.

These transformations are known by most systems like ObjectStore [12]. ObjectStore implements them by user-defined functions on a given class. The first transformation allows the migration of instances from a class to one of its subclass. The subtyping ensures that the migrated instance is always of the right type. The migration to a superclass is not authorized in OODBs leading to wrong typing of instances. These functions apply the transformations on the instances of the class. Default transformations are also provided by the OODB when automatic conversions can be deduced. In our catalog, we describe the different transformations on data that a primitive can undergo. In fact, the data transformations have to be expressed as dynamic integrity constraints on the instances of the new classes according to the old ones. In [7], the notion of integrity contraints is also extented in a similar sense.

Example of a primitive : add a class as a leaf

This primitive can lead to a conflict name between an inherited attribute and one of its attribute. The invariant 5 concerns the redefinition of attribute or method. In ObjectStore, since we can't redefine an attribute in a subclass, this invariant will be violated and the associated rule must be applied and suggests the deletion of the most specialized attribute. In addition, a transformation can be used in order to migrate instances of the superclass to the new class.

Based on an explicit definition of the notion of primitive, the catalog of primitives is a complete list of basic schema modifications, local to a class. We give for each primitive the different associated transformations, the appropriate invariants to check and the rules to apply in case of invariant violation. A primitive acts like a transaction : the process of evolution lets in a consistent state the schema and the database. We don't make any assertions on the semantic of the resulting object model. The definition of primitives covers the basic schema evolution functionnality that an OODB has to offer to be complete according to our definition of primitive. Certain systems don't offer all the primitive modifications that the underlying object model supports. For example, ORION restricts the change of the attribute type to a subtype only to simplify the evolution process. We will see in the paragraph 4 how we envisage to fill the gap.

\section{Three levels of complexity}

Our intent is to provide a complete classification of modifications for schema evolution. We propose three levels of modifications : primitive, composite and complex. Controlling the primitive level will help us to resolve modifications at higher levels of complexity. We propose to divide in two other categories the remaining set of modifications(See Figure 1). The composite modifications represent the intermediary level between primitive level and the most complex level ; they are rather simple but a little more complex than the primitive one. A given modification belongs to one and only one level. According to the definition of a primitive modification, the primitives are restricted in scope (restricted to one and only one concept) and simple (in that they embody one single type of modification applied to one single element of the concept at hand). This excludes modifications such as:

- transform a given class into one instance ; this example was given in [14] by Li and Mcleod ;

- produce different elements; e.g. split one class in several other classes;

- apply on different elements; e.g. coalesce several classes into one. These last two cases are proposed in the modifications taxonomy of [11].

In the first modification, instance is considered as a concept. We decided not to consider it as a concept for the definition of primitives. Otherwise, we have to consider data-driven modifications like Li and McLeod in [14]. We are purely schema concept-driven. The two last modifications involve one more than one element of a concept. Such modifications are 
categorized into the composite category of modifications, the intermediary level. We base our definition of composites on the notion of "link" between classes. Our intuition guides us to observe a "neighbourhood" of classes to discover modifications more complex than primitives. We see the schema as a net of classes linked together by inheritance, by the concept "Relationship" or by a client-supplier relation implemented with attributes of type "Reference on" a class or of type "Collection of references on" a class. We call respectively these three kinds of link: Inheritance, Relation and Reference.

\section{Definition}

Lets consider $n(n \geq 1)$ classes which are directly linked to a common class by one link of one of the three types: Inheritance, Relation, and Reference(regardless of the direction), these $(n+1)$ classes constitute a neighbourhood of classes, made up by 2 levels of classes ( 1 with the $\mathrm{n}$ classes and 1 with the common class); a composite modification can be one and only one of the three types of modifications:

- create one and only one level of a neighbourhood of classes in the schema

- remove one and only one level of a neighbourhood of classes from the schema

- change one and only one level of a neighbourhood of classes in the schema.

Symmetrically to the primitive definition, three kinds of modifications are included in this definition. We have underlaid in this definition that a composite is not a primitive; nevertheless, it can be any sequence of at least two primitives in accordance wih the definition. Create (resp. remove) a level means create (resp. remove) classes that make up a level of a neighbourhood. We can encounter several configurations of creation or removal of a level : classes of the level can be interior nodes or leaves according on the kind of link that it has been considered. The "change" category doesn't insert or delete a level of classes, but contains every modification that can be done on classes of a level. This includes changes on the "shape" of a level (i.d. the number of classes of the level can vary), changes on the nature of the link (i.d. an inheritance link can changed into a reference link) and, changes on the structure of the implied classes (i.d. migrate attributes on a Reference link). In general, a composite modification implies to change also the second level involved in the same neighbourhood of the level concerned by the modification (otherwise we wouldn't have in general any reason to make the evolution). A primitive was seen as an isolated modification of the schema. On the contrary, a composite defines a "context" of evolution : it results from an analysis of classes close together.

Example of a composite : merge sibling subclasses

This composite is part of the "change" category and involves to merge classes of one level to one class of the same level. In the semantic of this composite, the instances of the merged classes have to be hooked to the resulting class.

We have defined a catalog of the most typical and frequently occurring composite modifications for which we have found a decomposition in primitives. Such a decomposition proves that the associated composite can be applied and provides a way to make this evolution. We present the catalog of composites in the next paragraph. The complex level encompasses all the other modifications not included in the primitive and composites sets. The complex level represents the modifications which we don't control a priori. A complex can be rather simple -three primitives- or can be all kinds of mix of primitives and composites but is different from a composite. In the best of our knowledge, a complex can be decomposed in composites and primitives.

\section{Example of a complex :}

In a schema, a class Employee with subclasses EmployeePaidHourly, EmployeePaidMonthly, EmployeeOnComission represents an employee in a bank. The manager wants to change the semantic of Employee in order to easily transform the policy of payment of an employee without migrating instances of the Employee's subclasses. Conceptually, several actions has to be done :

- the class PaymentPolicy is created with its three corresponding subclasses HourlyPaymentPolicy, MonthlyPaymentPolicy and CommissionPaymentPolicy,

- the class Employee is linked now to PaymentPolicy by a Reference link.

- The subclasses of Employee are removed from the schema.

Physically, the instances of the Employee's subclasses have to be transformed into direct instances of Employee. For each of them, an instance of the PaymentPolicy's subclasses is created and the Reference link is established between both of these instancess. 
This modification is complex because it involves to create the PaymentPolicy's hierarchy (then two levels), to create a level between Employee and PaymentPolicy and to remove the level constituted by the Employee's subclasses. In addition, the transformation of instances is not direct because the initial semantic of Employee is scattered in two different hierarchy. We below describe the catalog of typical composites.

\section{Catalog of Composite Modifications}

We propose in the last paragraph a definition of composites that covers a broad set of modifications. Some of them are not very relevant, realistic and pertinent to schema evolution. The catalog of composites we present here concerns a subset of the composites. We have identified a catalog of 23 typical and frequent composites. We have differentiated Relation and Reference links. A relation between two classes can be modeled by a reference with lost of semantic. For this reason, some composites apply on both of the links. But, semantically, these two links are different and two different decompositions in primitives have to be provided. We support also to convert one link into the other one. In ObjectStore, the concept of relation can slow down the access of the objects ; to obtain a better performance, the manager can modify a relation to a simpler structure. A composite is described in the catalog by a name, a setting(input conditions), a description of the semantics and a decomposition in primitives. Such a decomposition validates what we have called in the introduction the applicability of a composite. We detail later this point.

Excerpt of the composite catalog : merge sibling sub-classes into one class

setting : sibling classes B and C (and more) might have common subclasses. Names of attributes of B and C have to be different. Any two attributes of B and C with identical names must have identical types. the B's and C's methods have their signatures different. $\mathrm{B}$ is the name of the resulting class.

semantics : migrate the instances of the evolving classes into the resulting class.

decomposition :

(1) change the target of inheritance link from the B's superclass to C with reclassification of C's instances into B's

(2) migrate-down C's attributes and methods to B

(3) delete class C's.

The figure 2 shows the different steps of this decomposition with the different extensions of $\mathrm{B}$ and $\mathrm{C}$ obtaining during evolution : ib, ic are the set of the original instances or the resulting instances of each primitive; ib' and ic' are the set of the old instances before the primitive, on which the transformations have been applied. The semantics implies to transform C's instances into B's. Thus, the primitive(1) is used with reclassification. The B's extension is augmented with the old C's instances. The modification(2) "empties" C of its contents to keep its semantics in B. This step is the "migrate" primitive applied as many attributes and methods as C contains. The B's instances are transformed if the system like ObjectStore considers one instance as sub-objects relative to the memebrship classe and its superclasses. During the deletion of $\mathrm{C}$ in the primitive(3), $\mathrm{C}$ has to be changed into $\mathrm{B}$ everywhere in the schema to ensure conceptual consistency. no transformation is needed since there is no risk to lose information.

If a primitive is not supported by an OODB, the primitive is solved like a composite and a decomposition has to be furnished in terms of the supported primitives but, conceptually, such a primitive remains at the primitive level even if it has been treated as a composite. There is some evidence that it exists a minimal set of primitives allowing the decomposition of the composites. In [6], a minimal set of basic modifications is discussed for the structural consistency of a schema. Then, if this primitive is used in a sequence of composites, it is replaced by its own sequence. The decompositions we propose in this catalog have been proved with the OODB ObjectStore [12].

Schema evolution, schema integration, and refactoring are concerned by the same problem of modifying an object model. We have studied this three domains to validate our catalog. Some of works we have studied are: schema evolution[2, 3, 11, 13, 14, 21], schema integration domain[8] and refactoring domain[17]. We have compared conceptually the modifications of these work with our composites in the catalog. We have not focused on the update of the instances. In [14], the instances are transformed in different ways than our modifications.

The following results give the comparisons between 44 modifications and our set of composites :

- exactly match : 26

- almost match : 14

- composite but not consider as typical : 3 
- new typical composite : 1 .

The 40 modifications that almost or exactly match cover 17 modifications on 23 composites of our catalog. With the criteria "almost match", few composites of the catalog are rather far from the compared modifications (more than 2 primitives of difference in the decomposition). Therefore, we decide to enhance three modifications of our catalog. On the 7 remaining composites of our catalog without comparison, 2 seem not typical and are suppressed; 1 is merged with an enhanced composite; the other 4 manage the Reference or Relationship link that we decide to keep. For example, a reference link can be transformed in a relationship link and vice-versa. It seems to us that these modifications are relevant to our catalogs because these two links, representing the notion of association in modeling, are important in database modeling and design and necessitate more attention in the problematic of evolution. We have rejected three composites in the studied work due to their simplicity or their rarety. A new typical composite concerns a modification that models an existing relation between two classes by a third class. This modifications enters in the category "Add a relation link". It seems to us that it can be useful when the manager desires to change the way to access the relation. The resulting catalog contains 21 typical composites, validated by the comparison study.

With these catalogs, we propose a process for schema evolution, solving the problems of applicability and conformity.

\section{Evolution process based on Catalogs}

Schema evolution leads to problems that we call the applicability and the conformity problems. The applicability problem can be formulated by two question : "is the solution applicable on the schema (the ODBS must be able to support this change) and how to apply the evolution?". The last question adresses the technical process of an evolution. The conformity problem refers to the unstability of the schema, the data and the application during the evolution. The schema evolution based on the catalogs can be expressed by a quick scenario of the evolution process that we will detail later. This scenario can be automated by a tool for which the catalogs are prerequisites. It is dedicated to schema evolution regardless of the way an evolution is done. At the moment, there are three ways to realize an evolution : by view [3, 20, 22], by version [16, 21] and effectively like in ObjectStore[12]. Ra and Rundensteiner[20] give the different views realizing primitives. The two first mechanisms achieve a virtual schema evolution: they allow to simulate a modification without the constraint of restructuring the database. Three major steps describe the process whose the actor is the schema manager :

1 - Request for modifications.

2- Identify the corresponding sequence of composites and/or primitives.

3- For any primitive :

- adjust the modification if the schema consistency is not preserved, otherwise,

- write transformations to update data if they don't have to be lost and,

- realize evolution (by view, by version or effectively).

The second step is complex because it supposes the correctness of the sequence. The sequence must carry out exactly the desired general modification, hopefully with acceptable performance considering time and memory. The challenge is hard and ambitious and our futur research will explore this area. For evolving the schema, a modification is carrying out and it acts like a transaction: after evolution, the schema is in a consistent state and the physical reorganization has been done correctly. The data have to be conform to the new class definitions. If rules of a primitive are not applied, the modification is rejected. In this case, the modification has to be reformulated in sequence and/or, probably, a primitive must be inserted to the sequence to "prepare" or to adjust the schema to be modified by this primitive. Sometimes, this situation is caused by a side-effect of the primitive on the conceptual reorganization that the manager had not predicted. However, if the schema consistency is preserved, the primitive can be applied to the schema and the conceptual reorganization has to be completed. Transformations of data are done on the physical reorganization in order to conform the data to their new structure: they must be transformed to a valid state according to the evolved schema. These transformations have to reorganize the data without loss of useful information. Depending on the primitive and the context of evolution, several transformations can be applied for only one primitive.

For each composite modification, the applicability is reached if every primitive of the sequence is supported by the OODB. The invariants have to be verified and the data updated for each primitive in the decomposition for the conformity problem. The sequence acts like a methodological process to update data in order not to lose information. We ensure that the applicability and conformity are solved if we apply sucessfully each primitive of the sequence. But, depending on detail 
implementations, certain primitive can be grouped together and solved together like a unit. This aspect is concerned by the optimization(in time and memory) of the sequence wich we are working on. For this problem, we have to study for example, at the physical level, which kind of update (immediate or lazy) uses, which sequence is optimal according to the clustering of data.

The evolution is seen at a higher degree of abstraction than the primitive level. An arbitrary modification(complex or composite) can be solved by the recognition of high level modifications: the composites, and completed by primitives. As it is inetresting to note in the excerpt of the catalog, the setting demands an ideal configuration of classes to ensure a minimum of rules violation. In fact, in reality, an evolution doesn't match exactly a composite or a primitive, and an adjustement has to be done. The first author, in a project on multimedia OODB [5, 9], has encountered a variant of one composite in a required evolution. With the catalogs, we ensure that a modification decomposed in a sequence of composite and primitive is applicable and we know how to solve the conformity problem by the primitive catalog. The tool we envision will support both of the two catalogs and will be able to apply a sequence of any known modifications. In fact, it would be very useful to implement a feature to recognize automatically known modifications inside an evolution, but it is a big challenge that demands form recognition. In addition, this tool should be incremental in the sense that a schema manager could be able to enhance the composite catalog with his own modifications as the primitive modifications that the underlying system doesn't offer.

\section{Ongoing and Future Work}

The level of primitive modifications that we have determined represents the atomic modifications that a system should offer by default to support the more complex modifications. We define two higher levels : composite and complex for which we propose a decomposition approach to solve them. We have designed a first version of the composite catalog according to a definition, containing the most typical and frequent composites. We want first to improve this catalog by providing an optimal(in time and memory) sequence of primitives for each composite. We are studying the different mechanisms of data update(lazy and immediate), the clustering etc. In addition, we would like to propose general guidelines for finding decomposition to ease the conceptual and physical conformity, which a schema manager could use to determine decompositions of his modifications. We have already many insights obtained by the catalog of composites. We plan to automate the decomposition process by a tool, in order to help the user during the schema evolution. We want to supply a rigorous approach for the process, based on algorithms for the correctness and the optimization of the sequence to "solve" any arbitrary modification. Solving means finding a decomposition of a modification into primitives or composites. In this sense, we think that we can provide assistance for the complex level and we would like to propose a semi-automated guidance for the decomposition into composites and primitives. In the future, we plan to study the recognition of known modifications into a desired schema evolution.

\section{Conclusion}

In the problematic of schema evolution, conceptual modifications are performed on the object model representing the schema. To solve any arbitrary modification, we adopt a decomposition approach at three levels of complexity, a key concept in software engineering: "real-life", complex schema modifications are solved by decomposition into simpler, well-known and controlled modifications, called composites, which in turn may be decomposed into so-called primitives. The lowest level is defined by the modifications called primitives for which we offer a comprehensive catalog based on a clear definition which is detailed in this paper. Our motivation was to present primitives as basic modifications with a minimal loss of data and as significant building blocks of the solutions for complex and composite modifications. On this basis, a catalog of the most typical and frequently occurring composites has been elaborated. It enhances the level of abstraction for schema evolution, furthers reuse, provides a virtual interface to the underlying database system . We have proved the decompositions of these composites with ObjectStore and we have validated it by comparing the composites with modifications discovered in the literature. This work is a prerequisite for effective tool support in order to provide automation of the two higher levels of modifications. The objective of this paper was to establish explicit definitions of the different modifications of a schema and to categorize them in catalogs in order to solve an arbitrary schema evolution. 


\section{References}

[1] T. Atwood, J. Duhl, G. Ferran, M. Loomis, and D. Wade. Object Database Standard: ODMG-93. Morgan Kaufmann Publishers, San Mateo, CA, 1994.

[2] Philippe Brèche, Fabrizio Ferrandina, Martin Kuklok. Simulation of schema change using views. In Proceedings of the 6th International Conference on Database and Expert Systems Applications ; pages 247-258, London, United Kingdom, September 1995.

[3] Elisa Bertino. A view mechanism fo object-oriented databases. In Proceedings of the International Conferenceon Extending Database Technology; pages 136-151, Vienna, Austria, March 1992.

[4] Jay Banerjee, Won Kim, Hyoung-Joo Kim, and Henry F. Korth. Semantics and implementation of schema evolution in objectoriented database systems. In Proceedings of the International Conference on Management of Data; pages 311-322, San Francisco, CA, May 1987.

[5] Gregor v. Bochmann, Brigitte Kerhervé, Abdelhakim Hafid, Petre Dini et, Anne Pons. Architectural design of adaptive multimedia systems. Workshop International IEEE "Multimedia Software Development", pages 31-40, Berlin, Allemagne, March 1996.

[6] Christine Delcourt, Roberto Zicari. The design of an integrity consistency checker (ICC) for an object-oriented database system. In Proceedings of the European Conference on Object-Oriented Programming, Geneva, Switzerland, July 1991.

[7] Anne Doucet, Stéphane Gançarski, Geneviève Jomier, Sophie Monties. Integrity Constraints and Versions. In the 6th International Workshop on Foundations of Models and Languages for Data and Objects "Integrity in Databases", Dagstuhl Castle, Germany, September 1996.

[8] Peter Fankhauser, Regina Motz, Gerald Huck. SIM Schema Inegration Methodology.Technical Report GMD/D4-4.1-V1.0, GMD-IPSI, Darmstadt, germany, February 1995.

[9] Brigitte Kerhervé, Anne Pons, Gregor v. Bochmann et, Abdelhakim Hafid. Metatada modelling for Quality of Service Management in distributed multimedia systems. In the first IEEE Metadata Conference, URL:http://www.nml.org/resources/misc/metatdata/proceedings/meta-home.html, Silver Spring, Maryland, USA, 16-18 April 1996.

[10] Alfons Kemper and Guido Moerkotte. Object-Oriented Database Management. Addison-Wesley, 1994.

[11] Won Kim. Introduction to object-oriented databases. The MIT Press, 1990. Computer Systems Series.

[12] Charles Lamb, Gordon Landis, Jack Orenstein, and Dan Weinreb. The ObjectStore database system. Communications of the ACM, 34(10):50-63, October 1991.

[13] B.S Lerner and A.N Habermann. Beyond schema evolution to database reorganization. In Proceedings of the Conference on Object-Oriented Programming: Systems, Languages and Applications, pages 67-76, Ottawa, Canada, October 1990.

[14] Qing Li and Dennis McLeod. Conceptual database evolution through learning. In Rajiv Gupta and Ellis Horowitz, editors, Object-Oriented Databases with Applications to Case, Networks and VLSI CAD, chapter 4, pages 62-74. PrenticeHall, Inc., 1991.

[15] Magdi M. A. Morsi, Shamkant B. Navathe, John Shilling. On behavioral schema evolution in object-oriented databases. In Proceedings of the 4th International Conference on extending Database technology, Cambridge, united Kingdom, March 1994.

[16] Erik Odberg. Schema versioning and class hierarchy modifications in object-oriented databases. In Workshop on supporting the Evolution of Class Definitions OOPSLA'93, Washington D.C., USA, 1993.

[17] William F.Opdyke. Refactoring object-oriented frameworks. Ph.D Thesis, University of Illinois at Urbana-Champaign, 1992.

[18] Anne Pons and Rudolf K. Keller. A catalog of primitive modifications for the conceptual evolution of object databases. Technical Report GELO-1006, Université de Montréal, Montreal, September 1995. 
[19] Anne Pons and Rudolf K. Keller. Evolving object database schemas by a catalog of primitive modifications. In Proceedings of Software Engineering and its Applications, Paris, France, Décembre 1995.

[20] Young-Gook Ra, Elke A. Rundensteiner. The MultiView OODB view system and implementation. Journal of Theory and Practice of Object systems, Special Issue on Subjectivity in Object-Oriented Systems(issue EDitors: Harold Ossher and William Harrison), John Wiley New York, 1996.

[21] John F. Roddick. A survey of schema versioning issues for database systems. Technical report UNISA TR CIS-94-010, Advanced Computer Research Centre, School of Computer and Information Science, University of South Australia, the Levels, Australia, 1994.

[22] Markus Tresch and Marc H. Scholl. Schema transformation without database reorganization. ACMSIGMOD Record, 22(1), March 1993.

[23] Emmanuel Waller. Schema updates and consistency. In Proceedings of the Second International Conference on Deductive and Object-Oriented Databases, pages 167-188, Munich, Germany, December 1991.

[24] Roberto Zicari. A framework for schema updates in an object-oriented database system. Technical Report 39-89, GIP Altair, Le Chesnay Cedex, France, October 1989.

[25] Roberto Zicari. A framework for schema updates in an object-oriented database system. In Proceedings of the Seventh International Conference on Data Engineering, pages 2-13, Kobe, Japan, April 1991.

Table 1: catalog of composites by name

\begin{tabular}{|c|c|c|c|}
\hline Type/Link $^{1}$ & Inheritance & Relation & Reference \\
\hline Add & $\begin{array}{l}\text { 1- create subclasses I and II } \\
\text { 2- conditional specialization } \\
\text { 3- create a common superclass } \\
\text { 4- promote attribute type to superclass }\end{array}$ & $\begin{array}{l}\text { 14- model ternary relation } \\
\text { with an existing relation }\end{array}$ & $\begin{array}{l}\text { 19- objectify attributes } \\
\text { 20- Join attributes into } \\
\text { collection }\end{array}$ \\
\hline Remove & $\begin{array}{l}\text { 5- merge into direct superclass } \\
6 \text { - merge into a new attribute of direct } \\
\text { superclass } \\
\text { 7- eliminate direct superclass } \\
\text { 8- convert superclass into attribute } \\
\text { type }\end{array}$ & $\begin{array}{l}\text { 15- deobjectify attributes } \\
\text { 16- expand relation }\end{array}$ & $\begin{array}{l}\text { 21- deobjectify attributes } \\
\text { 22- expand relation }\end{array}$ \\
\hline Change & $\begin{array}{l}\text { 9- merge sibling sub-classes } \\
10-\text { merge sibling superclasses into } \\
\text { one class } \\
11-\text { cut subclass into sibling classes } \\
12-\text { cut superclass into sibling classes } \\
13-\text { unit top classes }\end{array}$ & $\begin{array}{l}\text { 17- Relocate attribute I and } \\
\text { II } \\
18 \text { - transform relation into } \\
\text { collection }\end{array}$ & $\begin{array}{l}23 \text { - Relocate attribute I and } \\
\text { II } \\
24 \text { - tranform collection into } \\
\text { relation }\end{array}$ \\
\hline
\end{tabular}

\footnotetext{
${ }^{1}$. The modifications are classified in columns by the type of link : Inheritance, Relation and Reference; in rows by the kind of modifications: Add, Remove and Change.
} 
